\title{
Ansiedade, Depressão e Estresse do estudantado universitário pelo reinício das aulas em tempo de pandemia
}

Ansiedad, depresión y estrés del estudiantado universitario por la reanudación de clases en época de pandemia Anxiety, Depression and Stress of university students due to the resumption of classes in pandemic time

\section{Volumen 21, Número 3 \\ Setiembre - Diciembre}

pp. 1-22

\author{
Amândio Jamba Pedro da Fonseca \\ Ramiro Gross Tur \\ Anastácio Pascoal Epandi Canhanga \\ Maurício Firmino Morais
}

\section{Citar este documento según modelo APA}

Jamba, Amândio Pedro da Fonseca., Gross Tur, Ramiro., Canhanga, Anastácio Pascoal Epandi., e Morais, Maurício Firmino. (2021). Ansiedade, Depressão e Estresse do estudantado universitário pelo reinício das aulas em tempo de pandemia. Revista Actualidades Investigativas en Educación, 21(3), 1-22. Doi. 10.15517/aie.v21i3.46439 


\title{
Ansiedade, Depressão e Estresse do estudantado universitário pelo reinício das aulas em tempo de pandemia \\ Ansiedad, depresión y estrés del estudiantado universitario por la reanudación de clases en época de pandemia \\ Anxiety, Depression and Stress of university students due to the resumption of classes in pandemic time
}

\section{Amândio Jamba Pedro da Fonseca ${ }^{1}$ Ramiro Gross Tur ${ }^{2}$ Anastácio Pascoal Epandi Canhanga ${ }^{3}$ Maurício Firmino Morais ${ }^{4}$}

\begin{abstract}
Resumo: A pandemia da COVID-19 constitui ameaça à saúde humana, é identificada como um estressor significativo que coloca em risco a saúde psicológica e o bem-estar de pessoas em todo o mundo; pode desencadear níveis leves e graves de problemas psicossociais. O objetivo desta investigação foi descrever os níveis de ansiedade, depressão e estresse do estudantado do Instituto Superior Politécnico do Bié, no município do Kuito em Angola. Trata-se de um estudo quantitativo, não experimental, transversal com alcance descritivo. De forma não probabilística acidental, participaram 376 estudantes dos cursos de Comunicação Social, Contabilidade e Administração, Engenharia Informática e Psicologia, entre os quais 140 mulheres (37,23\%) e 236 homens (62,77\%), na faixa etária de 18 a 60 anos, com média de idade de 25,99 anos. A recolha de dados realizou-se no período de cinco a 30 de outubro de 2020 e a análíse fez-se durante os meses de janeiro e fevereiro de 2021. Aplicou-se a Escala de Ansiedade, Depressão e Stresse, EADS-21(Pais-Ribeiro, et al., 2004), é uma escala composta por três sub-escalas distribuidas em 21 itens que avaliam: Ansiedade, Depressão e Estresse, avaliados através de uma escala de resposta de tipo Likert, de 4 pontos. Os resultados mostram que o estudantado apresenta níveis baixos de Ansiedade, Depressão e Estresse. Conclui-se que, no sexo feminino, na faixa etária dos 18 aos 30 anos, nos primeiros anos de frequência e nos cursos de Contabilidade e Administração e Psicologia há maiores afetações dos indicadores Ansiedade, Depressão e Estress.
\end{abstract}

Palavras-Chave: efeitos psicológicos, pandemia, estudantes, educação superior.

\begin{abstract}
${ }^{1}$ Docente e Investigador do Instituto Superior Politécnico do Bié, Departamento de Psicologia, Kuito, Angola. Mestre em Educação Especial e Psicopedagogia. Direção electrónica: amandiojamba@gmail.com. ORCID: https://orcid.org/0000-0002-0135-2919

${ }^{2}$ Docente e Investigador da Universidad de Oriente, Departamento de Psicologia, Santiago de Cuba. Doutor em Ciências Pedagógicas. Direção electrónica: ramiro.gross@yahoo.com. ORCID: https://orcid.org/0000-0003-3892-7883
\end{abstract}

\begin{abstract}
${ }^{3}$ Docente e Investigador do Instituto Superior Politécnico do Bié, Departamento de Ciências de Base, Kuito, Angola. Mestre em Investigação em Matemáticas. Direção electrónica: pascoalkanhanga@hotmail.com. ORCID: https://orcid.org/0000-0003-3255-8087

${ }^{4}$ Docente do Instituto Superior Politécnico do Bié, Departamento de Psicologia, Kuito, Angola. Licenciado em Ciências da Educação, menção em Psicologia. Direção electrónica: mauriciomundjeli@gmail.com. ORCID: https://orcid.org/0000-0003-2817-6940
\end{abstract}

Artículo recibido: 26 de marzo, 2021

Enviado a corrección: 02 de julio, 2021

Aprobado: 23 de agosto, 2021 
Resumen: La pandemia del COVID-19 es una amenaza para la salud humana. Se ha identificado como un estresor significativo que pone en riesgo la salud psicológica y el bienestar de las personas en todo el mundo. Asimismo, puede desencadenar niveles leves y graves de problemas psicosociales. El objetivo de la presente investigación fue describir los niveles de ansiedad, depresión y estrés del estudiantado del Instituto Superior Politécnico del Bié en el municipio de Kuito en Angola. Se trata de un estudio cuantitativo, no experimental, transversal, de alcance descriptivo. De manera no probabilística y accidental, participaron 376 estudiantes de las carreras de Comunicación Social, Contabilidad y Administración, Ingeniería Informática y Psicología, de los cuales 140 son mujeres (37,23\%) y 236, hombres (62,77\%), de 18 a 60 años, con un promedio de edad de 25,99 años. La recolección de datos se realizó del 5 al 30 de octubre de 2020 y el análisis se efectuó durante los meses de enero y febrero de 2021. Se aplicó la Escala de Ansiedad, Depresión y Estrés, EADS-21 (País-Ribeiro, et al., 2004), que es una escala compuesta por tres subescalas distribuidas en 21 ítems que evalúan: Ansiedad, Depresión y Estrés, evaluados mediante una escala de respuesta tipo Likert, 4 puntos. Los resultados muestran que el estudiantado presenta bajos niveles de ansiedad, depresión y estrés. Se concluye que, en el sexo femenino, en el grupo etario de los 18 a los 30 años, en los primeros años de frecuencia y en las carreras de Contabilidad y Administración, y Psicología, existe mayores afectaciones de los indicadores Ansiedad, Depresión y Estrés.

Palabras clave: efectos psicológicos, pandemia, estudiantes, educación superior.

Abstract: The COVID-19 pandemic is a threat to human health, it is identified as a significant stressor that puts at risk the psychological health and well-being of people around the world; it can trigger mild and severe levels of psychosocial problems. The objective of this study was to describe the levels of anxiety, depression and stress of Higher Polytechnic Institute of Bié students in the municipality of Kuito in Angola. This is a quantitative, nonexperimental, cross-sectional study with a descriptive scope. With the non-probabilistic accidental criterion, 376 students participated from the Social Communication, Accounting and Administration, Computer Engineering and Psychology courses, participated 140 women (37.23\%) and 236 men (62.77\%), aged 18 to 60 years, with an average age of 25.99 years. The data collection was from October 5 to 30, 2020 and the analysis was during the months of January and February 2021. The Anxiety, Depression and Stress Scale, EADS-21 was applied (Pais-Ribeiro, et al., 2004), it is a scale composed by three sub-scales distributed in 21 items that evaluate: Anxiety, Depression and Stress, evaluated through a type response scale Likert, 4 points. The results show that students have low levels of Anxiety, Depression and Stress. It is concluded that, in females, aged between 18 and 30 years, in the first years of courses and in Accounting and Administration and Psychology courses, there are greater effects on the Anxiety, Depression and Stress indicators.

Keywords: psychological effects, pandemic, students, higher education.

\section{Introdução}

A pandemia de coronavírus 2019 (COVID-19), causada pelo SARS-CoV-2 (sigla inglesa - severe acute respiratory syndrome coronavirus 2), surgiu em Wuhan, capital da província de Hubei na China, no final de 2019; até ao início de 2020, havia se espalhado pelo mundo (Kamel-Abd e Raman-Rangan, 2020; Mazza et al., 2020).

Decorridos aproximadamente 14 meses após o seu início, até no dia 25 de fevereiro de 2021, foram confirmados quase 112.590 .567 casos em 192 países com mais de 2.498 .600 mortes certificadas (Johns Hopkins University, 2021). A tendência global progrediu para um aumento exponencial no número de casos; embora haja dinâmicas particulares que dependem da data do início da importação dos primeiros casos, das intervenções por cada país e das características sociodemográficas e económicas de cada contexto (Elías- Cuartas et al., 2020; Ho e Miethke-Morais, 2020). 
A rápida disseminação da doença, adicionada à falta de evidências científicas de tratamentos eficazes comprovados e a ausência de vacinas por se tratar de um vírus novo causou grande alarme mundial (Ochoa-Rosales, González-Jaramillo, Vera-Calzaretta e Franco, 2020). No final de janeiro de 2020, a Organização Mundial da Saúde declarou o surto da COVID-19 como uma emergência de saúde pública de interesse internacional e, no dia 11 de março do mesmo ano a enfermidade foi declarada pandemia (World Health Organization [WHO], 2020).

O surto da COVID-19 emergiu rapidamente como uma pandemia e, em curto período de tempo, causou ruptura nos sistemas económicos, perda de emprego, dificuldades nas relações interpessoais e isolamento social. Muitas pessoas experimentaram ansiedade, depressão e estresse como uma resposta natural à adversidade. Diversos estudos estão a ser realizados em quase todo mundo para descobrir uma vacina para COVID-19, mas o seu impacto na saúde mental permanece em grande parte pouco estudado (Arslan, Yıldırım, Tanhan, Buluş e Allen, 2020).

No continente africano, o primeiro caso da COVID-19 registou-se no Egito no dia 14 de Fevereiro de 2020, importado da China por ser o principal parceiro comercial da África; portanto, existem grandes volumes de viagens que facilitaram a chegada do vírus nesta região (Gilbert et al., 2020). Até ao dia 25 de fevereiro de 2021, a África contava com 3.856.581 casos positivos, com 102.470 mortes e 3.421 .526 recuperações, onde a África do Sul e o Egipto se destacam como os países mais afetados (Centres for Diseases Control and Prevention [CDC], 2021).

Em Angola, o primeiro caso da COVID-19 registou-se na província de Luanda, capital deste país no dia 21 de Março de 2020, importado de Portugal; no final do mês de Junho registraram-se os primeiros dois casos fora de Luanda, isto na província do Cuanza-Norte e, até ao dia 25 de fevereiro de 2021 o país contava com 20.640 casos confirmados, 501 falecimentos e 19.235 recuperados; importa salientar que, nos últimos dias, o país tem estado a presenciar um aumento exponencial de casos positivos (CDC, 2021; Johns Hopkins University, 2021).

Devido à sua extrema virulência, várias medidas têm sido usadas pelos governos de todos os países afetados, como por exemplo, o encerramento das fronteiras e a quarentena, no sentido de diminuir a curva de contaminação e evitar colapsos dos sistemas de saúde para permitir que recebam assistência médica o maior número possível de pessoas infectadas (Wu e McGoogan, 2020). Neste sentido, Fawaz e Samaha (2020), definem a quarentena como o 
isolamento e proibição da livre circulação ou limitação das viagens de indivíduos para evitar e/ou diminuir a chance de contaminação a uma infecção ou doença grave que possam estar submetidos.

Na mesma linha de ideias, Ho e Mieth-keMorais (2020), assinalam que o isolamento social visa a redução da transmissibilidade entre pessoas com consequente achatamento da curva de contaminação, contudo, não impede a transmissão da infecção por si só, enquanto o vírus estiver a circulação na comunidade. Ainda assim, a medida de isolamento é importante para preparar os sistemas de saúde e permitir que esses prestem assistência suficiente e adequada aos enfermos.

Por outro lado, deu-se o reinício das aulas no dia cinco de outubro de 2020, em todas instituições do Ensino Secundário e Superior em Angola. No entanto, registam-se algumas incertezas com relação ao sucesso das atividades académicas devido ao aumento de casos positivos da COVID-19 no país. Por este facto, se reconhece a necessidade de formular o seguinte problema de investigação: Quais são os níveis de ansiedade, depressão e estresse do estudantado do Instituto Superior Politécnico do Bié?

Torna-se importante desenvolver investigações nesta área, uma vez que, a literatura consultada apoia a visão de que, o isolamento imposto como medida de prevenção á pandemia da Covid- 19, tem um enorme impacto em muitos aspectos da vida das pessoas, causa consideráveis tensões psicológicas que desencadeiam uma variedade de problemas psicológicos. Por esta razão, o presente estudo tem como objetivo descrever os níveis de ansiedade, depressão e estresse do estudantado do Instituto Superior Politécnico do Bié.

\section{Referencial teórico}

\subsection{Covid-19 desde a perspectiva psicossocial}

Desde a perspectiva psicossocial, uma pandemia constitui um evento que ativa respostas psicológicas coletivas que incidem seu avanço ou contenção, assim como possível geração de respostas emocionais de inquietação ou desordem social (Taylor, 2019).

Assim mesmo, a COVID-19 é uma ameaça à saúde humana identificada como um estressor significativo que coloca em risco a saúde psicológica e bem-estar de pessoas em todo o mundo. Este estressor pode desencadear níveis leves e graves de problemas psicossociais, como ansiedade, depressão, transtornos de estresse pós-traumático, angústia, medo, desespero, sentimento de frustração e tédio, pensamentos obsessivo-compulsivos, fadiga laboral entre profissionais de saúde, inflexibilidade psicológica, problemas 
comportamentais e consequências negativas associadas aos excessivos tempos na internet (Çiçek, Tanhan, Bulus e Arslan, 2020; Liu et al., 2020; Mucci, Mucci e Diolaiuti, 2020).

Na mesma linha de ideias, Liang et al. (2020), afirmam que, esses tipos de epidemias levam os indivíduos a experimentarem problemas psicológicos como transtorno de estresse pós-traumático, sofrimento psicológico, depressão e ansiedade.

Estes transtornos são caracterizados por preocupações intensas e persistentes que ocasionam tensão, inquietação e dificuldades de concentrar-se. Causam sofrimento, muita sobrecarga psíquica ao indivíduo, o que o torna propenso ao adoecimento mental que, por sua vez, repercute-se diretamente no desempenho das atividades diárias (Lima Francisco, Barros, Pacheco, Nardi, e Alves, 2020; Vilchez-Cornejo et al., 2020).

\subsection{Ansiedade}

A ansiedade constitui um dos fatores mais preocupantes que afetam o sucesso académico do estudantado de todos os subsistemas de ensino. Caracteriza-se por tristeza, excitação "preocupação/angústia" causado pelo deslocamento de medo e esperança (Aydın e Tiryaki, 2017). No mesmo sentido, na opinião de Tuncel et al. (2020), a ansiedade é um dos mais graves problemas do tempo atual, faz com que seus efeitos sejam sentidos em muitas questões, especialmente na realização de trabalhos académicos, o que coloca em risco o desempenho do estudantado.

Os transtornos de ansiedade geralmente começam em uma idade precoce, com o período de maior risco de emergência entre 10-25 anos, e os sintomas ocorrem antes dos 35 anos em $80-90 \%$ dos casos (Tanhan, Karaman e Nalbant, 2020). Para o estudantado universitário, este é um período muito importante, em que se observam níveis e sintomas de ansiedade elevados.

Desde o ponto de vista conceptual, os transtornos de ansiedade englobam transtornos que compartilham características de medo excessivo, ansiedade e perturbações relacionadas aos comportamentos. Medo é a resposta emocional perante uma iminente ameaça real ou percebida, enquanto ansiedade é a antecipação de ameaça futura (American Psychiatric Association [APA], 2014).

Nesta perspectiva, Tanhan, Karaman e Nalbant (2020), afirmam que a ansiedade é uma condição que resulta em reações físicas, como aperto de coração, palpitações cardíacas, tremor, dor de cabeça ou suor causada por emoções de medo, decorrentes da sensação de um indivíduo de estar sendo ameaçado em várias situações. 
Por outro lado, Tuncer e Yilmaz (2020), assinalam que, é errôneo perceber a ansiedade como um significado totalmente negativo, pois que, quando é mantida sob controle, em nível moderado desempenha um papel significativo no processo de aprendizagem e tem uma característica que estimula positivamente o indivíduo.

\subsection{Estresse educacional}

Na última década, os estudos sobre o estresse educacional têm ganhado espaço em vários países, comunidades científicas e faz parte de nossas vidas e nos afeta tanto mental quanto fisicamente (Tuncay, Müdüroğlu e Bulut, 2020). Desde o ponto de vista conceptual, Kuyumcu (2020), define o estresse educacional como à conexão de pressão ao estudo, carga de trabalho e preocupação com notas.

Para Hamdan Abdala e Elnadeef (2019), o estresse é um estado de excitação física e psicológica produzido por estressores internos ou externos que são percebidos pelo indivíduo como um desafio e que excede sua capacidade ou recursos para enfrentar.

Nesta perspectiva, Niemi e Vainiomäki (2006), ressaltam que, o estresse tem muitos efeitos negativos no estudantado e as pessoas reagem de maneira diferente sob diferentes fatores estressantes; Personalidades, e estratégias de aprendizagem diferentes podem afetar o estresse acadêmico experimentado pelo estudantado.

As tensões associadas às cargas horárias e assimilação de novos regulamentos são descritos na literatura psicológica como fatores estressantes e têm grande influência no ajuste e rendimento académico por parte do estudantado (Tian, McClain, Moore e Lloyd, 2019). Na mesma opinião, Shields, Hunnell, Tucker e Price (2020), afirmam que aumento do estresse no estudantado universitário tem uma correlação positiva com os níveis de depressão e ansiedade que experimentam.

Os efeitos psicológicos derivados do estresse têm um impacto na saúde emocional e mental do indivíduo; tais efeitos muitas vezes se manifestam através dos transtornos do humor, como depressão, ansiedade, transtorno bipolar, problemas cognitivos (de pensamento), mudanças de personalidade e comportamentos problemáticos (Shields, et al., 2020). 


\subsection{Depressão}

De acordo com a APA (2018), a depressão é uma doença médica comum, tratável que afeta negativamente a maneira de pensar, agir, sentir e causa sentimentos de tristeza e / ou perda de interesse nas atividades.

A depressão é um transtorno que se caracteriza pela presença de humor triste, vazio ou irritável, acompanhado de alterações físicas e cognitivas que afetam significativamente a capacidade de funcionamento do indivíduo (APA, 2014).

Segundo Kivrak e Altin (2019), a depressão pode originar uma variedade de problemas emocionais e físicos e diminui a capacidade da pessoa para funcionar no local de trabalho e em casa.

Os sintomas da depressão podem variar de leves a graves e podem incluir: desde sentirse triste até estar deprimido; perda de interesse ou prazer em atividades antes apreciadas; mudanças no apetite - perda ou ganho de peso não relacionado à dieta; problemas para dormir ou dormir muito; perda de energia ou aumento da fadiga; aumento da atividade física sem propósito (por exemplo, torcer as mãos ou andar de um lado para o outro); sentir-se sem valor ou culpado; dificuldade em pensar, concentrar-se ou tomar decisões; pensamentos de morte ou suicídio (APA, 2018).

Por outro lado, Kivrak e Altin (2019) relatam que ficar triste não é o mesmo que ter depressão; o processo de luto é natural e único para cada indivíduo e compartilha algumas das mesmas características de depressão. Tanto o luto quanto a depressão podem envolver tristeza intensa e retraimento das atividades usuais.

\section{Metodologia}

\subsection{Enfoque}

Trata-se de um estudo quantitativo, não experimental, transversal com alcance descritivo, dado que, analisou-se o fenómeno em suas condições naturais, sem alterar nem manipular a variável; o fenómeno selecionado como objeto de estudo observou-se em um momento único de tempo (Hernández-Sampieri e Torres, 2018).

\subsection{Unidades de análise}

Participaram 376 estudantes dos cursos de Comunicação Social, Contabilidade e Administração, Engenharia Informática e Psicologia, entre os quais houve 140 mulheres $(37,23 \%)$ e 236 homens $(62,77 \%)$, na faixa etária de 18 a 60 anos, com média de idade de 
25,99 anos (ver tabela 1). O critério de amostragem foi o não probabilístico acidental, visto que, foi selecionado o estudantado que apareceu nas primeiras quatro semanas do reinício das aulas (Cossio-Bolaños, 2015).

Foram considerados os seguintes critérios:

Inclusão: Estudantes matriculados para o primeiro semestre do ano 2020 no Instituto Superior Politécnico do Bié, estudantes que apareceram nas primeiras quatro semanas de aulas, estudantes que decidiram participar voluntariamente na investigação por meio da assinatura do consentimento informado, estudantes que responderam totalmente 0 instrumento apresentado.

Exclusão: Estudantes que não apresentaram disponibilidade para participar na investigação, estudantes que não tenham efetuado matrículas para o primeiro semestre de 2020, estudantes que assinaram o consentimento informado, mas que, decidiram não participar na investigação, estudantes que não responderam totalmente o instrumento aplicado.

O alunado selecionado foi solicitado através das assinaturas dos consentimentos informados. Aplicou-se o questionário nas salas de aulas, em uma única sessão, no momento de intervalo, para não afetar o normal desenvolvimento das tarefas académicas, os participantes precisaram um período de tempo de aproximadamente 20 minutos em média para completar o questionário e utilizou-se o método tradicional de lápis e papel. A recolha de dados realizou-se de cinco a 30 de outubro de 2020 e a análise da informação decorreu entre janeiro e fevereiro de 2021. 


\begin{tabular}{|c|c|c|c|}
\hline \multicolumn{2}{|c|}{ Variáveis Sociodemográficas } & $\mathrm{Fi}$ & \multirow{2}{*}{$\begin{array}{c}\% \\
62,77\end{array}$} \\
\hline Sexo & Homens & 236 & \\
\hline & Mulheres & 140 & 37,23 \\
\hline \multirow[t]{2}{*}{ Idade } & 18 a 30 anos & 316 & 84,04 \\
\hline & 31 a 60 anos & 60 & 15,96 \\
\hline \multirow[t]{4}{*}{ Curso } & Comunicação Social & 45 & 11,97 \\
\hline & Contabilidade e Administração & 167 & 44,41 \\
\hline & Engenharia Informática & 36 & 9,57 \\
\hline & Psicologia & 128 & 34,04 \\
\hline \multirow[t]{5}{*}{ Ano de frequência } & $1^{\circ}$ Ano & 156 & 41,49 \\
\hline & $2^{\circ}$ Ano & 77 & 20,48 \\
\hline & $3^{\circ} \mathrm{Ano}$ & 87 & 23,14 \\
\hline & $4^{\circ} \mathrm{Ano}$ & 42 & 11,17 \\
\hline & $5^{\circ}$ Ano & 14 & 3,72 \\
\hline \multirow[t]{2}{*}{ Período } & Regular & 211 & 56,12 \\
\hline & Pós-Laboral & 165 & 43,88 \\
\hline
\end{tabular}

Fonte: Elaboração Própria (2021)

\subsection{Técnica de coleta de dados}

Para a recolha de dados, aplicou-se a EADS-21(Pais-Ribeiro, et al., 2004). A escala original está em Inglês e denomina-se DASS-21 Depression, Anxiety and Stress Scale. Foi adaptada e validada para a Língua Portuguesa pelo grupo de investigadores de Pais-Ribeiro, Honrado e Leal (2004). É uma escala composta por três sub-escalas que avaliam: Ansiedade, Depressão e Estresse. A sub-escala de depressão avalia sintomas, como inércia; anedonia; disforia; falta de interesse/envolvimento; auto-depreciação; desvalorização da vida e desânimo. A de ansiedade excitação do sistema nervoso autónomo; efeitos musculoesqueléticos; ansiedade situacional; experiências subjetivas de ansiedade. Por fim, a sub-escala de estresse avalia dificuldade em relaxar; excitação nervosa; fácil perturbação/ agitação; irritabilidade/reação exagerada e impaciência.

A EADS-21 é dirigida a indivíduos maiores de 17 anos de idade e está constituída por 21 itens, agrupados em três sub-escalas, formadas por sete itens cada, avaliados através de uma escala de resposta de tipo Likert, de quatro pontos, que reenviam para a severidade e frequência dos sintomas experimentados nos últimos sete dias - "na semana passada" (0 "não se aplicou nada a mim", 1 - "aplicou-se a mim algumas vezes", 2 - "aplicou-se a mim muitas vezes", e 3 - "aplicou-se a mim a maior parte das vezes"). A cotação dá-se pela soma dos resultados dos sete itens, obtendo-se uma nota para cada sub-escala com um resultado 
mínimo de 0 e máximo de 21. Desta maneira, notas mais elevadas correspondem a estados afetivos mais negativos. Com relação a consistência interna, os resultados obtidos por PaisRibeiro et al. (2004) demonstram o alfa de Cronbach de 0,80 como média das três sub-escalas.

$\mathrm{Na}$ presente investigação, para a adaptação e aplicação do instrumento no contexto angolano, teve-se em conta a validez de conteúdo pelo critério de expertos aplicando o coeficiente de concordância de Kendall (W), cujos resultados mostraram uma correlação positiva $(W=0,71$. Sig. = 0,05); e analisou-se também a consistência interna, onde encontrouse o alfa de Cronbach de 0,894 nesta população.

\subsection{Análise de dados}

Após a obtenção dos dados, foram utilizadas as planilhas do Excel 2016 e o Statistical Package for Social Sciences (SPSS-24.0), para obter as medidas de tendência central e variabilidade média (M), desvio padrão (DP), frequência (Fi) e percentagem (\%). Fez-se a análise descritiva da variável de acordo com a idade, sexo, curso, ano de frequência e período.

O presente estudo foi aprovado pelo Comité de Ética ( $\mathrm{ad} \mathrm{hoc)} \mathrm{do} \mathrm{Instituto} \mathrm{Superior}$ Politécnico do Bié.

\section{Resultados}

Com relação ao indicador Ansiedade, verifica-se que a população estudada apresenta uma média de 3,65 em um total de 14 pontos, com um desvio padrão de 3,72. Desta maneira, esta amostra de participantes apresenta níveis baixos de Ansiedade em comparação ao ponto médio da escala (7 pontos). Nesta variável se observa que a população masculina obteve uma média $(M=3,20)$ inferior à apresentada pela população feminina $(M=4,09)$.

No indicador Depressão, os resultados mostram uma média de 4,57 com um desvio padrão de 4,24. Nesta varável se observa que, ainda os resultados estão por baixo do nível médio da escala (7), estão mais próximos deste ponto do que do valor zero (0) e tendo em consideração o desvio padrão, presume-se que o indicador encontra-se parcialmente afetado nesta população. Enquanto aos resultados deste indicador segundo o género, aprecia-se que as médias são bastante similares.

Com respeito ao indicador Estresse, a população estudada mostrou uma média de 5,34 e um desvio padrão de 4,15. Estes resultados mostram níveis baixos de estresse, já que, as médias estão por baixo do ponto médio da escala, tal como aconteceu nos resultados da variável depressão. Ao considerar o valor da média e do desvio padrão, também infere-se que 
o indicador estresse está relativamente afetado na amostra estudada, por enquanto, estes valores indicam relativa proximidade ao ponto médio da escala. Enquanto às diferenças entre homens e mulheres, verifica-se que, o género feminino apresenta uma média $(M=5,76)$ superior à apresentada pelo género masculino $(M=4,91)$, conforme se observa na tabela 2 .

Tabela 2. Valores por Média e Desvivos-Padrão de Ansiedade, Depressão e Estresse do estudantado do Instituto Superior Politécnico do Bié, Kuito-Angola, 2020-2021

\section{Segundo sexo}

\begin{tabular}{|l|c|c|c|c|}
\hline \multicolumn{1}{|c}{ Indicadores } & \multicolumn{2}{c|}{ Masculino } & \multicolumn{2}{c|}{ Feminino } \\
\hline Ansiedade & M & DP & M & DP \\
\hline Depressão & 3,20 & 3,43 & 4,09 & 4,00 \\
\hline Estresse & 4,46 & 4,20 & 4,68 & 4,27 \\
\hline
\end{tabular}

Nota: M=Média; DP= Desvio Padrão.

Fonte: Elaboração própria (2021)

A variável idade foi dividida em duas faixas (18-30 e 31-60). Os resultados mostram que, no indicador Ansiedade, o estudantado na faixa etária de 18-30 anos apresenta uma média de 3,44 e os da faixa maior de 31 apresentam a média de 1,50. Assim, estes participantes manifestam níveis de Ansiedade que são bastante inferiores ao ponto médio da escala. Também se observa que os níveis de ansiedade são superiores no estudantado de menor idade com relação aos de maior idade.

No indicador Depressão, o estudantado na faixa de 18-30 anos apresentam a média de 4,27 e os maiores de 30 apresentam 1,77 como média. Ao analisar o nível de depressão nos menores de 30 anos compreende-se que, o valor da média $(M=4,27)$ é inferior ao ponto médio da escala, se se analisa o desvio padrão ( $\mathrm{DP}=4,14)$, pode-se inferir que a Depressão foi relativamente afetada nesta faixa etária. Em contrapartida, o estudantado da faixa etária superior aos 30 anos apresenta níveis muito baixos, o que indica que a situação pandémica e o reinício das aulas não lhes têm afetado muito.

Com relação ao indicador Estresse, os menores de 30 anos apresentam a média de 5,01 e os maiores de 30 apresentam a média de 1,77. Estes dados indicam que, embora ambas as faixas etárias manifestem níveis inferiores ao ponto médio, o grupo na faixa de 18 a 30 anos mostra valores próximos ao ponto médio, por isso, infere-se que, têm sido moderadamente afetados; a diferença dos maiores de 30 anos, que parecem não ter sofrido afetações no indicador Estresse. (Ver tabela 3). 
Tabela 3. Valores por Média e Desvivos-Padrão de Ansiedade, Depressão e Estresse do estudantado do Instituto Superior Politécnico do Bié, Kuito-Angola, 2020-2021 Segundo idade

\begin{tabular}{|l|c|c|c|c|}
\hline \multicolumn{1}{|c}{ Indicadores } & \multicolumn{3}{c}{$18-30$} & \multicolumn{3}{c|}{$31-60$} \\
\hline Ansiedade & $\mathrm{M}$ & $\mathrm{DP}$ & $\mathrm{M}$ & $\mathrm{DP}$ \\
\hline Depressão & 3,44 & 3,65 & 1,50 & 2,69 \\
\hline Estresse & 4,27 & 4,14 & 1,77 & 2,50 \\
\hline
\end{tabular}

Nota: $M=$ Média; $\mathrm{DP}=$ Desvio Padrão.

Fonte: Elaboração própria (2021)

Os participantes encontram-se divididos em quatro cursos (Comunicação Social, Contabilidade e Administração, Engenharia Informática e Psicologia). Neste sentido, no Indicador Ansiedade observou-se a média de 3,05 para o estudantado de Comunicação Social, 3,48 para os de Contabilidade e Administração, 3,03 para os de Engenharia Informática e 3,51 para os de Psicologia. Assim, estas médias manifestam níveis de Ansiedade por baixo do ponto médio, o que permite deduzir que este indicador não tem afetado de maneira notável ao estudantado estudado.

Relativamente à Depressão, o estudantado de Comunicação Social apresenta a média de 3,68, os de Contabilidade e Administração 5,02, os de Engenharia Informática 3,17 e os de Psicologia 3,82. Estes resultados indicam níveis baixos de Depressão. Sem embargo, chamam atenção os resultados obtidos no curso de Contabilidade e Administração, onde a média $(M=5,02)$ e o desvio padrão ( $D P=4,35)$, o que sugerem que, o estudantado deste curso tem mostrado mais afetações no indicador Depressão do que o estudantado de outros cursos.

No indicador Estresse, o estudantado de Comunicação Social apresenta uma média de 4,0, os de Contabilidade e Administração 5,15, os de Engenharia Informática 4,33 e os de Psicologia 5,11. Estes resultados demostram níveis baixos de estresse, conforme o ponto médio da escala; No entanto, a sua proximidade ao valor do ponto médio da escala, e os valores do desvio padrão, pode-se compreender que o indicador Estresse resulta relativamente afetado. Também se observa que os cursos de Contabilidade e Administração e Psicologia são os que apresentam os valores de estresse mais elevados. (Ver tabela 4). 
Tabela 4. Valores por Média e Desvivos-Padrão de Ansiedade, Depressão e Estresse do estudantado do Instituto Superior Politécnico do Bié, Kuito-Angola, 2020-2021

\section{Segundo curso}

\begin{tabular}{|l|c|c|c|c|c|c|c|c|}
\hline \multicolumn{1}{|c|}{ Indicadores } & \multicolumn{2}{c}{$\begin{array}{c}\text { Comunicação } \\
\text { Social }\end{array}$} & \multicolumn{2}{c}{$\begin{array}{c}\text { Contabilidade e } \\
\text { Administraça }\end{array}$} & \multicolumn{2}{c|}{$\begin{array}{c}\text { Engenharia } \\
\text { Informática }\end{array}$} & \multicolumn{2}{c|}{ Psicologia } \\
\cline { 2 - 10 } & M & DP & M & DP & M & DP & M & DP \\
\hline Ansiedade & 3,05 & 3,62 & 3,48 & 3,33 & 3,03 & 3,86 & 3,51 & 3,76 \\
\hline Depressão & 3,68 & 3,91 & 5,02 & 4,35 & 3,17 & 3,95 & 3,82 & 3,67 \\
\hline Estresse & 4,00 & 3,36 & 5,15 & 3,78 & 4,33 & 4,71 & 5,11 & 4,13 \\
\hline
\end{tabular}

Nota: $\mathrm{M}=$ Média; $\mathrm{DP}=$ Desvio Padrão.

Fonte: Elaboração própria (2021)

No que concerne ao indicador Ansiedade, o estudantado do primeiro ano obteve a média de 3,72 , os do segundo 3,84 , os do terceiro 3,63 , os do quarto 1,60 e os do quinto 1,50. Estes resultados manifestam níveis baixos de Ansiedade em sentido geral. No entanto, na análise destes dados aprecia-se uma notável diferença entre os resultados do estudantado que frequenta desde o primeiro até o terceiro ano e o que frequenta o quarto e o quinto anos dos seus respectivos cursos, já que, os grupos estudantis dos anos finais apresentam menores níveis de Ansiedade.

No indicador Depressão, o estudantado do primeiro ano apresenta a média de 5,07 , os do segundo ano 3,95 , os do terceiro 4,55 , os do quarto 2,38 e os do quinto 3,00 . Tais médias representam níveis baixos de Depressão. Sem embargo, se identifica que o estudantado do primeiro e terceiro anos apresenta médias próximas ao ponto médio da escala. Estes dados indicam que estes anos de frequência são os mais afetados pela depressão perante o reinício das aulas em contexto da pandemia da Covid-19.

Relativamente ao Estresse, os discentes do primeiro ano obtiveram a média de 5,52, os do segundo 4,66 , os do terceiro 5,26 , os do quarto 3,12 e os do quinto 3,64 . Estes resultados manifestam níveis de Estresse por baixo do ponto médio da escala. No entanto, se observa que o estudantado que frequenta desde o primeiro até o terceiro anos é o mais afetado pelo indicador estresse, em comparação com os estudantes dos anos terminais.

A análise das variáveis Ansiedade, Estresse e Depressão com relação aos anos de frequência permite compreender que o estudantado dos três primeiros anos de frequência mostra maior nível de afetação emocional com relação aos discentes dos anos terminais. (Ver tabela 5). 
Tabela 5. Valores por Média e Desvios-Padrão de Ansiedade, Depressão e Estresse do estudantado do Instituto Superior Politécnico do Bié, Kuito-Angola, 2020-2021 segundo ano de frequência

\begin{tabular}{|l|c|c|c|c|c|c|c|c|c|c|}
\hline Indicadores & \multicolumn{2}{c}{$\mathbf{1}^{\circ}$ Ano } & \multicolumn{2}{c}{$\mathbf{2}^{\circ}$ Ano } & \multicolumn{2}{c}{$3^{\circ}$ Ano } & \multicolumn{2}{c|}{$4^{\circ}$ Ano } & \multicolumn{2}{c|}{$5^{\circ}$ Ano } \\
\hline & M & DP & M & DP & M & DP & M & DP & M & DP \\
\hline Ansiedade & 3,72 & 3,69 & 3,84 & 3,89 & 3,63 & 3,37 & 1,60 & 2,62 & 1,50 & 1,55 \\
\hline Depressão & 5,07 & 4,52 & 3,95 & 3,79 & 4,55 & 3,96 & 2,38 & 2,98 & 3,00 & 2,00 \\
\hline Estresse & 5,52 & 4,32 & 4,66 & 3,84 & 5,26 & 3,36 & 3,12 & 4,14 & 3,64 & 3,08 \\
\hline
\end{tabular}

Nota: $M=$ Média; $\mathrm{DP}=$ Desvio Padrão.

Fonte: Elaboração própria (2021)

No indicador Ansiedade, o estudantado do período regular obteve a média de 3,45 e o do pós-laboral 3,53, o que indica níveis baixos de Ansiedade e não se apreciam grandes diferenças no comportamento desta variável entre os períodos de aulas dos discentes.

Com relação ao indicador Depressão, o estudantado do período regular apresenta a média de 4,70 e os do pós-laboral 4,04. Estes resultados indicam níveis baixos de Depressão e uma ligeira superioridade no nível de Depressão do estudantado do período regular com ralação ao do pós-laboral.

Finalmente, no que se refere ao Estresse, o estudantado do regular obteve a média de 5,35 e os do pós-laboral 4,67. Ainda que os resultados indiquem níveis baixos, é válido ressaltar que esses valores mostram certa proximidade ao ponto médio da escala, pelo que, pode-se compreender que, todo o estudantado manifesta algumas sensações de estresse (Ver tabela 6).

Tabela 6. Valores por Média e Desvivos-Padrão de Ansiedade, Depressão e Estresse do estudantado do Instituto Superior Politécnico do Bié, Kuito-Angola, 2020-2021 Segundo período

\begin{tabular}{|l|c|c|c|c|}
\hline \multicolumn{1}{|l|}{ Indicadores } & \multicolumn{2}{c}{ Regular } & \multicolumn{2}{c|}{ Pós-Laboral } \\
\hline Ansiedade & M & DP & M & DP \\
\hline Depressão & 3,45 & 3,50 & 3,53 & 3,87 \\
\hline Estresse & 4,70 & 4,27 & 4,04 & 3,97 \\
\hline
\end{tabular}

Nota: M=Média; DP= Desvio Padrão.

Fonte: Elaboração própria (2021) 


\section{Discussão}

Este estudo objetivou descrever os níveis de ansiedade, depressão e estresse do estudantado do Instituto Superior Politécnico do Bié.

Sabe-se que o estresse positivo prepara-nos para a ação, pode nos ajudar a lidar com situações que, talvez poderiam salvar nossas vidas, o que torna necessário, ter em mente, que sua manifestação psicofisiológica pode transbordar se as pessoas estiverem sujeitas à situações que causam altos níveis de estresse durante muito tempo e, ainda mais, se estiverem ligadas à mídia e / ou redes sociais que apresentam notícias que possam gerar maior medo ou desinformação ao público (Marquina, 2020).

De acordo aos resultados descritivos por sexo nos indicadores Ansiedade, Depressão e Estresse, todo o estudantado apresenta níveis baixos em comparação ao ponto médio da escala. Embora não se aplicaram provas estatísticas que permitissem identificar diferenças significativas nestas variáveis com relação ao sexo, os dados mostram que o sexo feminino teve maiores afetações nestes indicadores. Isto pode estar ligado às características psicológicas das mulheres, que mostram-se mais preocupadas e expressam maior sentimento de autocuidado e conservação. Estes resultados mostram semelhança com os achados por Marquina (2020), quem expõe que as mulheres apresentam maiores níveis de estresse em comparação com os homens.

Sem embargo, em um estudo publicado por Carneiro Pinto, Martins, Pinheiro e Oliveira (2015) sobre Ansiedade, Depressão e Estresse dos jovens adultos e adultos portugueses, os autores não identificaram diferenças na depressão, estresse e ansiedade em função do sexo, idade, ou formação académica.

No mesmo sentido, os autores Alves Apóstolo, Tanner e Lee Arfken (2012), encontraram resultados que concordam com os do presente estudo, uma vez que, estes autores descrevem que as mulheres obtiveram pontuações significantemente mais altas do que os homens nos indicadores avaliados.

Resultados diferentes foram encontrados por Ozamiz-Etxebarria, Dosil-Santamaría, Picaza-Gorrochategui e Idoiaga-Mondragon (2020), estes autores ressaltam que, a partir do confinamento, a população tem apresentado níveis altos de sintomatologia no que se refere a ansiedade, depressão e estresse.

Referente à idade, os resultados do presente estudo relatam que, o estudantado de todas as faixas etárias apresenta níveis considerados baixos nestes indicadores. A pesar de não se constatarem diferenças estatisticamente significativas nos indicadores depressão, 
ansiedade e estresse, pôde-se revelar que os mais jovens mostraram médias mais altas do que o estudantado de maior idade; Estes resultados são similares aos encontrados por Orellana e Orellana (2020); Ozamiz-Etxebarria, et al. (2020), pois que, estes autores assinalam que, a população mais jovem apresenta maiores níveis de ansiedade e estresse.

Os resultados por curso manifestam que, todo o estudantado apresenta níveis considerados baixos nos indicadores ansiedade, depressão e estresse. Somente se encontrou uma ligeira diferença no indicador estresse, onde os cursos de Contabilidade e Administração e o de Psicologia mostraram médias superiores com relação às dos outros cursos.

Os resultados encontrados neste estudo salientam que o estudantado apresenta níveis baixos nos indicadores ansiedade, depressão e estresse. Embora não se contrastou estatisticamente, pode-se apreciar que o estudantado dos três primeiros anos de frequência mostrou maiores médias nos indicadores analisados do que os discentes dos anos terminais. Estes resultados contrapõem - se aos encontrados por Rebelo-Pinto, Amaral, Silva, Silva, Leal e Paiva (2012), os autores encontraram diferenças entre os alunos do $3^{\circ}$ ciclo e os alunos do ensino secundário, com resultados mais favoráveis aos alunos mais jovens.

De acordo com os resultados por período, encontrou-se que, todo o estudantado apresenta níveis baixos nos indicadores estudados. Embora não se revelam diferenças estatisticamente significativas entre os períodos; pôde-se apreciar nas médias dos indicadores de depressão e estresse, valores ligeiramente superiores nos discentes do período regular com relação aos do pós-laboral.

\section{Conclusões}

Este estudo é o primeiro que aborda esta temática nesta região do país e, apresenta resultados relevantes sobre Ansiedade, Depressão e Estresse do estudantado do Instituto Superior Politécnico do Bié, no município do Kuito em Angola. Estes resultados poderão contribuir para que a classe estudantil saiba sobre seus estados psicológicos nas dimensões estudadas. Oferecerá um conjunto de pressupostos teóricos e práticos que podem facilitar o Estado angolano e a direção do Instituto Superior Politécnico do Bié, em particular, em adotar medidas de biossegurança á COVID-19 que coincidam com as reais necessidades psicológicas do estudantado e que possam salvaguardar seus estados psicológicos para o sucesso académico desejado.

Todavia, existem algumas limitações que devem ser consideradas no momento de interpretar estes resultados. Não estão representados todos os cursos que conformam o 
Instituto, pelo facto de que, nem todo o estudantado aceitou participar do estudo; a falta de controlo de algumas variáveis sociodemográficas, como a zona de residência e o local de trabalho, que são elementos que podem contribuir para alterações dos estados psíquicos do estudantado; A falta da utilização de uma prova estatística que pudesse revelar as diferenças significativas na análise das variáveis estudadas.

Sugere-se continuar com investigações nesta temática em outras instituições de Angola, a fim de ter uma visão mais global para reforçar ou contradizer aos resultados desta pesquisa.

A pandemia da COVID-19 constitui ameaça à saúde humana, é identificada como um estressor significativo que coloca em risco a saúde psicológica e o bem-estar de pessoas em todo o mundo; pode desencadear níveis leves e graves de problemas psicossociais, como ansiedade, depressão, transtornos de estresse pós-traumático, angústia, medo, desespero, sentimento de frustração e tédio, pensamentos obsessivos-compulsivos, fadiga laboral entre profissionais de saúde, inflexibilidade psicológica e problemas comportamentais.

O estudantado do Instituto Superior Politécnico do Bié apresenta níveis baixos de Ansiedade, Depressão e Estresse. Embora não se constatou a partir da utilização de provas estatísticas, as médias mostram que, existem ligeiras diferenças no comportamento das variáveis, assim, revelou-se que, no sexo feminino, na faixa etária dos 18 aos 30 anos, nos primeiros anos de frequência e nos cursos de Contabilidade e Administração e Psicologia há maiores afetações dos indicadores Ansiedade, Depressão e Estresse.

\section{Referências}

Alves Apóstolo, João Luís., Allen Tanner, Barry., e Lee Arfken, Cynthia. (2012). Análise fatorial confirmatória da versão portuguesa da Depression Anxiety Stress Scale-21. Revista Latino-Americana de Enfermagem, 20(3), 1-7. doi: https://doi.org/10.1590/S010411692012000300022

American Psychiatric Association. (2014). Diagnostic and statistical manual of mental disorders (DSM-5). Estados Unidos: American Psychiatric Pub.

American Psychiatric Association. (2018). The american psychiatric association practice guideline for the treatment of patients with schizophrenia. Estados Unidos: American Psychiatric Pub.

Arslan, Gökmen., Yıldırım, Murat., Tanhan, Ahmet., Buluş, Metin., and Allen, Kelly-Ann. (2020). Coronavirus Stress, Optimism-Pessimism, Psychological Inflexibility, and Psychological Health: sychometric Properties of the Coronavirus Stress Measure. International Journal of Mental Health and Addiction. doi: https://doi.org/10.1007/s11469-020-00337-6 
Aydın, Aytaç., and Tiryaki, Sebahattin. (2017). A study on the determination of the factors affecting the anxiety level of university students (KTU sample). Kastamonu University Journal of Forestry Faculty, 17(4), 715-722. doi: https://doi.org/10.17475/kastorman.282559

Carneiro Pinto, Joana., Martins, Patrícia., Burm Pinheiro, Teresa., e Cardoso Oliveira, Ana. (2015). Ansiedade, Depressão e Stresse: Um Estudo com Jovens Adultos e Adultos Portugueses. Psicologia, Saúde \& Doenças, 16(2), 148-163. doi: http://dx.doi.org/10.15309/15psd160202

Centres for Diseases Control and Prevention. (25 $5^{\text {th }}$ February, 2021). Africa CDC updates on COVID-19. Recuperado de https://africacdc.org/covid-19/

Cossio-Bolaños, Marco António. (2015). Métodos de investigación cuantitativa en Ciencias de la Educación. Talca, Chile: Universidad Católica del Maule.

Çiçek, İlhan., Tanhan, Ahmet., Bulus, Metin., e Arslan, Gökmen. (2020). Psychological inflexibility predicts depression and anxiety during COVID-19: acceptance and commitment therapy perspective. Journal of Health and Social Behavior, 24, 386-396.

Elías-Cuartas, Daniel., Arango-Londoño, David., Guzmán-Escarria, Gissel., Muñoz, Edgar., Caicedo, Diana., Ortega-Lenis, Delia. ... e Méndez, Fabián. (2020). Análisis espaciotemporal del SARS-coV-2 en Cali, Colombia. Revista de Salud Pública, 22(2), 1-6. doi: https://doi.org/10.15446/rsap.V22n2.86431

Fawaz, Mirna., and Samaha, Ali. (2020). COVID-19 quarantine: Post-traumatic stress symptomatology among Lebanese citizens. International Journal of Social Psychiatry, 66(7), 664-674. doi: https://doi.org/10.1177/0020764020932207

Gilbert, Marius., Pullano, Giulia., Pinotti, Francesco., Valdano, Eugenio., Poletto, Chiara. Boëlle, Pierre-Yves. ... Colizza, Vittoria. (2020). Preparedness and vulnerability of African countries against importations of COVID-19: a modelling study. Lancet, 395(10227), 870-877. doi: https://doi.org/10.1016/S0140-6736(20)30411-6

Hamdan Abdala, Ayman Hamad Elneil., e Elsadig Elnadeef, Elsadig Ali. (2019). English Language Anxiety and Stress among Saudi Students in the First Year at College of Sciences and Arts in Dharan Aljanoob. International Journal of Linguistics, Literature and Translation (IJLLT), 2(1), 270-275. doi: https://doi.org/10.32996/ij|lt.2019.2.1.33

Hernández-Sampieri, Roberto., y Mendoza Torres, Christian Paulina. (2018). Metodología de la Investigación Científica: Las rutas cuantitativa, cualitativa y mixta. México: McGrawHill.

Ho, Yeh-Li., e Miethke-Morais, Anna. (2020). COVID-19: o que aprendemos? Journal Brasileiro de Pneumologia, 46(3), 1-2. Recuperado de https://www.scielo.br/j/jbpneu/a/BKjJPMMf3rrjkFVnZdmTBGF/?format=pdf\&lang=pt 
Johns Hopkins University. (2021). Coronavirus COVID-19 Global Cases by the Center for System Science and Engineering (CSSE). Recuperado de https://coronavirus.jhu.edu/map.html

Kamel-Abd, Rawaa., e Raman-Rangan, Vinoth. (2020). Assessment of the implementation of preventive measures by Iraqis people to reduce the spread of COVID-19 pandemic. Revista de Salud Pública, 22(2), 1-5. doi: https://doi.org/10.15446/rsap.V22n2.86475

Kivrak, Ali Osman., e Altin, Mehmet. (2019). A Scrutiny on the Changes in the Self-Respect, Anxiety, and Depression Levels of the University Students Performing Individual and Team Sports. International Journal of Higher Education, 8(3), 117-125. doi: https://doi.org/10.5430/ijhe.v8n3p117

Liang, Leilei., Ren, Hui., Cao, Ruilin., Hu, Yueyang., Qin, Zeying., Li, Chuanen., e Mei, Songli. (2020). The Effect of COVID-19 on Youth Mental Health. Psychiatric Quarterly, 91. doi: https://doi.org/10.1007/s11126-020-09744-3

Lima Francisco, Leilane Camila Ferreira., Correia Barros, Alice., Pacheco, Mariana da Silva., Egidio Nardi, Antonio., e Medeiros Alves, Verônica. (2020). Ansiedade em minorias sexuais e de gênero: uma revisão integrativa. Journal Brasileiro de Psiquiatria, 69(1), 4856. doi: https://doi.org/10.1590/0047-2085000000255

Liu, Shuai., Yang, Lulu., Zhang, Chenxi., Xiang, Yu-Tao., Liu, Zhongchun., Hu, Shaohua., and Zhang, Bin. (2020). Online mental health services in China during the COVID-19 outbreak. The Lancet Psychiatry, 7(4). doi: https://doi.org/10.1016/S22150366(20)30077-8

Marquina Lujan, Jesus Romano. (2020). Autopercepción del estrés en aislamiento social en tiempos de Covid-19. Revista ConCiencia EPG, 5(1), 85-99. doi: https://doi.org/10.32654/CONCIENCIAEPG.5 -1.6

Mazza, Cristina., Ricci, Eleonora., Biondi, Silvia., Colasanti, Marco., Ferracuti, Stefano., Napoli, Christian., and Roma, Paolo. (2020). A Nationwide Survey of Psychological Distress among Italian People during the COVID-19 Pandemic: Immediate Psychological Responses and Associated Factors. International Journal of Environmental Research and Public Health, 17(9), 1-14. doi: https://doi.org/10.3390/ijerph17093165

Mucci, Federico., Mucci, Nicola., e Diolaiuti, Francesca. (2020). Lockdown and isolation: psychological aspects of COVID-19 pandemic in the general population. Clinical Neuropsychiatry, 17(2), 63-64. doi: https://doi.org/10.36131/CN20200205

Niemi, Päivi Mirja-Liisa., e Vainiomäki, Paula. (2006). Medical students' distress - quality, continuity and gender differences during a six-year medical programme. Medical Teacher, 28(2), 136-141. doi: https://doi.org/10.1080/01421590600607088

Ochoa-Rosales, Carolina., González-Jaramillo, Nathalia., Vera-Calzaretta, Aldo., e Franco, Oscar. (2020). Impacto de diferentes medidas de mitigación en el curso de la pandemia de COVID-19 en Chile: proyección preliminar para el período del 14 de abril al 14 de mayo. Revista de Salud Pública, 22(2), 1-6. doi: https://doi.org/10.15446/rsap.V22n2.86380 
Orellana, Carlos Iván., e Orellana, Ligia Maria. (2020). Predictores de síntomas emocionales durante la cuarentena domiciliar por pandemia de COVID19 en EI Salvador. Actualidades en Psicología, 34(128), 103-120. doi: http://dx.doi.org/10.15517/ap.v34i128.41431

Ozamiz-Etxebarria, Naiara., Dosil-Santamaría, Maria., Picaza-Gorrochategui, Maitane., e Idoiaga-Mondragon, Nahia. (2020). Niveles de estrés, ansiedad y depresión en la primera fase del brote del COVID-19 en una muestra recogida en el norte de España. Cuadernos de Saúde Pública, 36(4). doi: https://doi.org/10.1590/0102-311X00054020

Pais-Ribeiro, Jose., Honrado, Ana., e Leal, Isabel. (2004). Contributos para o estudo da adaptação portuguesa das escalas de Ansiedade, Depressão e Stress (EADS) de 21 itens de Lovibond e Lovibond. Psicologia, Saúde \& Doenças, 5, 229-239. Recuperado de https://www.redalyc.org/pdf/362/36250207.pdf

Rebelo-Pinto, Teresa., Amaral, Cátia., Neves da Silva, Vera., Silva, Joana., Leal, Isabel., e Paiva, Teresa. (2012). Hábitos de sono e ansiedade, depressão e stress: que relação? Em L. Mata, F. Peixoto, J. Morgado, J.C. Silva, e V. Monteiro (Eds.), $12 .{ }^{\circ}$ Colóquio Psicologia Educação, Aprendizagem e Desenvolvimento: Olhares Contemporâneos através da Investigação e da Prática (pp. 990-1006). Brasil: ISPA - Instituto Universitário.

Shields, Margaret., Hunnell, William., Tucker, Melanie., and Price, Annie. (2020). Building Blocks and Coloring Away Stress: Utilizing LEGO and Coloring as Stress Reduction Strategies among University Students. Journal of Health Education Teaching, 11(1), 2431. Recuperado de https://eric.ed.gov/?id=EJ1268742

Tanhan, Ahmet., Karaman, Mehmet Akif., and Nalbant, Ahmet. (2020). The Effect of Counseling on Anxiety Level from the Perspective of Ecological Systems Theory: A Quasi-experimental Pre-test - Post-test Control Group Study. International Journal of Psychology and Educational Studies, 7(3), 58-69. doi: https://doi.org/10.17220/ijpes.2020.03.006

Taylor, Steven. (2019). The Psychology of Pandemics: Preparing for the Next Global Outbreak of Infectious Disease. Estados Unidos: Cambridge Scholars Publishing.

Tian, Lu., McClain, Shannon., Moore, Marisa., e Lloyd, Howard. (2019). An Examination of Ethnic Identity, Self Compassion, and Acculturative Stress in Asian International Students. Journal of International Students, 9(2), 635-660. doi: https://doi.org/10.32674/jis.v9i2.617

Tuncay, Nazime., Müdüroğlu, Ruhsan., e Bulut, Ayşe. (2020). Educational stress, social stress and gender differences among university students. Journal of Educational and Instructional Studies In The World, 10(2), 37- 46. Recuperado de https://www.researchgate.net/publication/341272473 EDUCATIONAL STRESS SOCI AL STRESS AND GENDER DIFFERENCES AMONG UNIVERSITY STUDENTS

Tuncel, Filiz., Yapıcı, Assim., Akman, Pınar., Elçi, Abdullah Cemil., Demirogları, Burcu., and Kutlu, Mahmut Oğuz. (2020). Foreign language anxiety of adolescent students. African Educational Research Journal, 8(2), S164-S169. doi: https://doi.org/10.30918/AERJ.8S2.20.044 
Tuncer, Murat., e Yilmaz, Ömer. (2020). Relations Attitude Towards Mathematics Lessons: Anxiety and Academic Success. REDIMAT - Journal of Research in Mathematics Education, 9(2), 173-195. doi: https://doi.org/10.17583/redimat.2020.4061

Vilchez-Cornejo, Jennifer., Viera-Morón, Ronald David., Larico-Calla, Gabriel., Alvarez-Cutipa, Daniela Carla., Sánchez-Vicente, Juan., Taminche-Canayo, Ruth. e ToroHuamanchumo, Carlos. (2020). Depression and Abuse During Medical Internships in Peruvian Hospitals. Revista Colombiana de Psiquiatría, 49(2), 76-83. Recuperado de http://www.scielo.org.co/scielo.php?script=sci arttext\&pid=S0034-74502020000200076

World Health Organization. (11 March, 2020). Coronavirus disease 2019 (COVID-19): Situation Report - 51. Recuperado de https://coronavirus.jhu.edu/map.html

Wu, Zunyou., e McGoogan, Jennifer. (2020). Characteristics of and important lessons from the coronavirus disease 2019 (COVID-19) outbreak in China: Summary of a report of 72314 cases from the Chinese Center for Disease Control and Prevention. Journal of the American Medical Association, 323(13), 1239-1242. doi: https://doi.org/10.1001/jama.2020.2648 
Revista indizada en

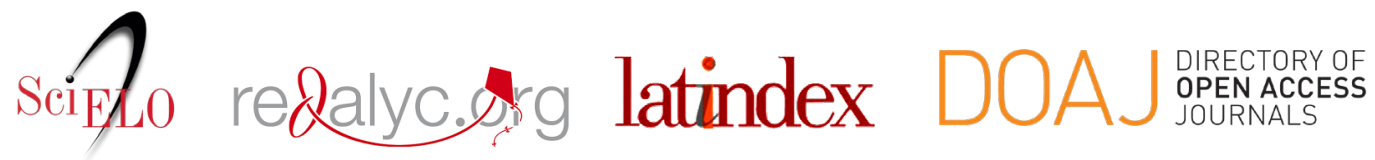

Distribuida en las bases de datos:

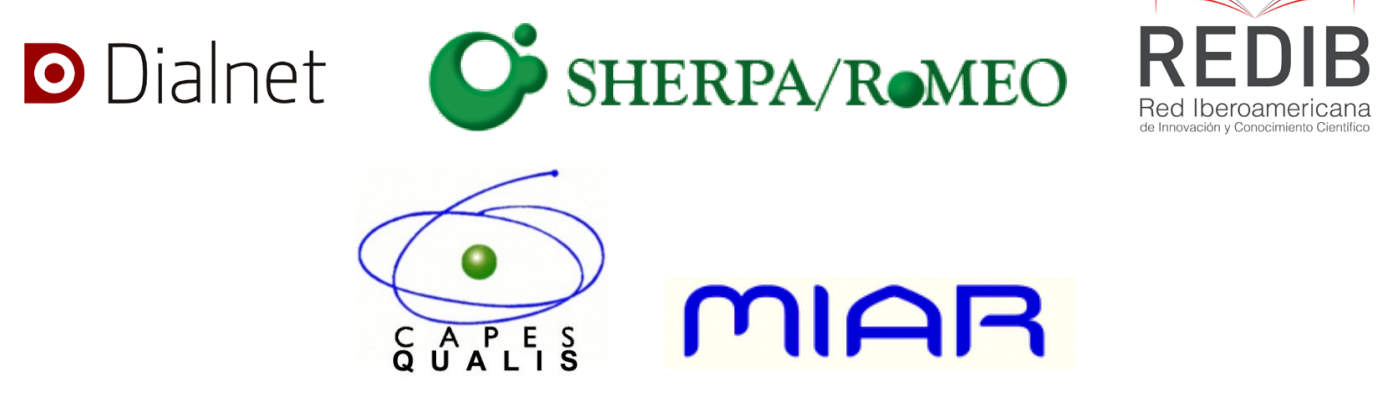

